\title{
BMJ
}

\section{Patients' experiences of screening for type 2 diabetes: prospective qualitative study embedded in the ADDITION (Cambridge) randomised controlled trial}

\author{
Helen Eborall, post-doctoral research fellow, ${ }^{1}$ Richard Davies, director of general practice studies, ${ }^{1}$ Ann- \\ Louise Kinmonth, professor of general practice, Simon Griffin, programme leader, ${ }^{2}$ Julia Lawton, senior \\ research fellow ${ }^{3}$
}

$\overline{{ }^{1} \text { General Practice and Primary Care }}$ Research Unit, Department of Public Health and Primary Care, University of Cambridge,

Cambridge CB2 OSR

${ }^{2}$ MRC Epidemiology Unit, Strangeways Research Laboratory, Cambridge CB1 8RN

${ }^{3}$ Research Unit in Health, Behaviour and Change, School of Clinical Sciences and Community Health, The University of Edinburgh, Medical School, Edinburgh EH8 9AG

Correspondence to: $\mathrm{H}$ Eborall hce21@medschl.cam.ac.uk

doi:10.1136/bmj.39308.392176.BE

\section{ABSTRACT}

Objectives To provide insight into factors that contribute to the anxiety reported in a quantitative study of the psychological effect of screening for type 2 diabetes. To explore expectations of and reactions to the screening experience of patients with positive, negative, and intermediate results.

Design Prospective qualitative interview study of patients attending a screening programme for type 2 diabetes.

Setting Seven general practices in the ADDITION (Cambridge) trial in the east of England.

Participants 23 participants (aged 50-69) attending different stages in the screening process.

Results Participants' perceptions changed as they progressed through the screening programme; the stepwise process seemed to help them adjust psychologically. The first screening test was typically considered unimportant and was attended with no thought about its implications. By the final diagnostic test, type 2 diabetes was considered a strong possibility, albeit a "mild" form. After diagnosis, people with screen detected type 2 diabetes tended to downplay its importance and talked confidently about their plans to control it. Participants with intermediate results seemed uncertain about their diagnosis, and those who screened negative were largely unaware of their remaining high risk. Conclusions This study helps in understanding the limited psychological impact of screening for type 2 diabetes quantified previously, in particular by the quantitative substudy of ADDITION (Cambridge). The findings have implications for implementing such a screening programme in terms of timing and content.

\section{INTRODUCTION}

Type 2 diabetes mellitus is a progressive disease, which can lead to considerable morbidity and mortality as a result of cardiovascular, renal, and retinal complications. Disease onset may occur up to 12 years before clinical diagnosis so many patients are asymptomatic. ${ }^{1}$ Screening by measuring blood glucose concentrations can diagnose type 2 diabetes and identify people with impaired fasting glucose or impaired glucose tolerance who are at risk of developing the condition. ${ }^{2}$ Evidence suggests that earlier detection and treatment may lead to improved health outcomes, ${ }^{3}$ and that behavioural and drug interventions in people with impaired glucose tolerance can reduce progression to type 2 diabetes. ${ }^{4}$ However, it is not clear whether the potential population benefits outweigh the possible costs, which include adverse psychological effects of screening and subsequent treatment.

The Anglo-Danish-Dutch study of intensive treatment in people with screen detected diabetes in primary care $^{5}$ (the ADDITION trial) is evaluating the cost effectiveness of screening and intensive treatment of screen detected cases. A substudy ${ }^{6}$ of the ADDITION (Cambridge) trial investigating the psychological impact of screening reported minimal adverse effect overall—no significant differences were found between the screening and control participants on psychological measures. People who screened positive at the first test reported a significantly greater psychological effect than those who screened negative, but effects were small and mean scores were not clinically relevant. ${ }^{6}$ At three to five months (after participants had completed the screening process) and 12-15 months, the more tests a participant had before screening negative, the more they worried about developing diabetes. However, levels of worry were low and effect sizes small. ${ }^{6}$

Recent qualitative research has highlighted considerable diversity in the emotional reactions of people diagnosed with type 2 diabetes through routine testing (compared with those diagnosed after illness) ${ }^{78}$ and a lack of understanding of their risk of cardiovascular disease. $^{9}$ Only one qualitative study has explored screening for type 2 diabetes from patients' perspectives. ${ }^{10}$ This highlighted a lack of understanding of the meaning of raised blood glucose. Few diagnosed patients thought their diabetes was a potentially severe condition, and those who received negative results (but were still at high risk) reported reassurance and no plans to change their lifestyle. This study was limited by a retrospective design, which could not 
capture the temporal relation between patients' expectations and experiences of the screening process or which aspects of screening may have fostered the low levels of anxiety observed. Furthermore, it only looked at people with positive or negative results, not those with intermediate results (impaired glucose tolerance or impaired fasting glucose).

We devised a prospective qualitative study to provide insight into the factors that contribute to anxiety during screening, as noted in the quantitative psychological impact substudy of ADDITION (Cambridge), and to provide insight into expectations and reactions to the screening experience of patients with positive, negative, and intermediate results.

\section{METHOD}

Our qualitative approach enabled us to identify themes during data collection, rather than test predetermined hypotheses. ${ }^{11}$ We incorporated a prospective component to capture participants' experiences and views at different stages during the screening process and to explore whether and how these changed after receiving their results.

\section{Participants}

We were keen to capture the experience of patients through the entire stepwise screening process (box 1). Because only $7 \%$ of people in ADDITION (Cambridge) who took the first test (random blood glucose) went on to take the final test (oral glucose tolerance) we sampled at three stages:

(1) We began by sampling at the point of referral for the final test; all patients with oral glucose tolerance tests scheduled during a defined period were invited for interview $(n=65)$. The resulting sample comprised 13 participants who were interviewed both before this test and again after receiving their test results.

(2) A second group of participants $(n=21)$ was purposively invited for interview after their final test results to redress the uneven balance of sex and diagnosis achieved in the first group. The resulting sample comprised five participants who were interviewed once (after test results).

(3) To capture the views of participants at the initial test (random blood glucose), we invited all patients attending for tests during one practice session $(\mathrm{n}=15)$. The resulting sample comprised five participants who were interviewed once, shortly after their test.

All participants were patients at seven ADDITION (Cambridge) study practices; recruitment was by

\begin{tabular}{lcccc}
\hline Final diagnosis of participants in a trial of screening for diabetes & $\begin{array}{c}\text { Impaired glucose } \\
\text { tolerance or impaired } \\
\text { fasting glucose }\end{array}$ & Negative & $\begin{array}{c}\text { Negative (random or } \\
\text { fasting blood glucose } \\
\text { only) }\end{array}$ \\
Sex & Type 2 diabetes & 6 & 1 & 2 \\
Male & 5 & 1 & 2 & 3 \\
\hline Female & 3 & & \\
\hline
\end{tabular}

Box 1| The ADDITION (Cambridge) trial screening programme

A stepwise screening procedure in people aged 40-69 years without known diabetes. People identified in the top quartile of risk of having prevalent undiagnosed type 2 diabetes by automated search of medical records ${ }^{12}$ are invited by letter to attend their local general practice for screening

Screening procedure

Visit 1: random whole blood (capillary) glucose and glycosylated haemoglobin tests

- If random blood glucose 5.5-11.0 mmol// proceed to visit 2

- If random blood glucose $\geq 11.1 \mathrm{mmol} / \mathrm{l}$ proceed to visit 3

Visit 2: fasting whole blood (capillary) glucose

- If fasting blood glucose $\geq 6.1 \mathrm{mmol} / \mathrm{l}$ or fasting blood glucose 5.5-6.0 mmol/l and glycosylated haemoglobin (from visit 1 ) $\geq 6.1 \%$ proceed to visit 3

Visit 3 at hospital outpatient centre: oral glucose tolerance test ${ }^{13}$ and clinical, anthropometric, and biochemical measures

- Diagnosis of type 2 diabetes was made according to the World Health Organization criteria ${ }^{2}$

- A second (confirmatory) oral glucose tolerance test was needed if a patient had tested $5.5-11.0 \mathrm{mmol} / \mathrm{l}$ at random blood glucose, 5.5-6.0 mmol/l at fasting blood glucose, and glycosylated haemoglobin $\geq 6.1 \%$

- Results were faxed to the patient's general practitioner for discussion with patients

Mean timing of (interview study) participants' tests:

- Day 1 Invitation sent to patient

- Day 20 Attend random blood glucose

- Day 47 Attend fasting blood glucose

- Day 91 Attend oral glucose tolerance test

invitation letter with an opt-in reply slip. The table provides a breakdown of participants' sex and diagnosis.

\section{Data collection}

HE conducted all interviews in participants' homes or workplaces. All patients gave written consent. Interviews covered the different stages of the screening process (box 2). The initial question, "Thinking back to when you received the invitation to screening what were your initial feelings?" encouraged participants to tell their story of the screening experience from the beginning. Interviews were audio recorded and transcribed verbatim.

\section{Analysis}

The analysis was informed by grounded theory, involving concurrent data collection and analysis, together with systematic efforts to check and refine emerging categories of data. ${ }^{1415}$ Themes were not predetermined; rather, those that emerged in early interviews were discussed in team meetings $(\mathrm{HE}, \mathrm{RD}, \mathrm{JL})$ and 
Box 2 Topic guide for interviews

Before oral glucose tolerance test

Invitation letter

Reactions to being invited for screening and reasons for attending

Perceptions of personal susceptibility to diabetes

Random blood glucose test

Expectations of test

Perceptions and understanding of test result and its implications

Fasting blood glucose test

Expectations of test

Perceptions and understanding of test result and its implications

Oral glucose tolerance test

Expectation about test

Anticipated result

Type 2 diabetes

Awareness and understanding of the disease

Perceived seriousness of the disease

Ideas about the causes of the disease

After results of the oral glucose tolerance test

Oral glucose tolerance test and diagnosis

Reflections on expectations of the test

Perceptions and understanding of test result

Emotional reactions to the result

If result negative or impaired glucose tolerance

Perceptions of future disease susceptibility

Intention to implement lifestyle changes

Emotional reactions

If diagnosed with type 2 diabetes

Perceptions of the disease (including perceived seriousness)

Emotional reactions

Views about information and advice from health professionals

Intention to implement lifestyle changes

Screening programme

Good and bad aspects of testing procedures

Views about timing, location, and information provided

were used to inform subsequent interviews and analysis. Throughout the interview and analysis phases, team meetings focused on exploring patients' underlying reasoning, discussing deviant cases, and reaching agreement on recurrent themes and findings. $\mathrm{HE}$ and $\mathrm{RD}$ independently read through and cross compared all transcripts. We used NVivo7 (QSR International), a qualitative data indexing package, to help in data coding and retrieval.

\section{RESULTS}

Participants' accounts of their screening experience showed how their perceptions of type 2 diabetes and their own personal risk changed over the course of the stepwise screening programme (box 1). Participants seemed to undergo a process of psychological adjustment, typically from attending the first screening test without considering its implications, to the final test where they confronted the possibility of having diabetes, as demonstrated by these contrasting quotes:

Patient 5 (male, impaired glucose tolerance, age 69) "I wasn't concerned at all, you know I thought, well I'll just go along and if I can help well okay all well and good, go and see what happens."

Patient 18 (female, impaired glucose tolerance, age 64) "So I go for number one, I go for number two and then I have to go to number three. So it's a build up all the time, making me think, well OK there's a possibility you know ... there's a strong possibility you know' in that sense [. . .] you've gone through the three, so your brain's adjusted anyway."

\section{Initial stages of the screening process}

Participants' reflections on the initial ADDITION invitation letter highlighted an unquestioning perception of screening being "good" (box 3). Most participants seemed to have considered the initial test "routine" and thought little about the implications of the possible results, an attitude typified by one patient's comment that "it can only be a good thing." Attenders rarely expected to test positive, except for one woman who had a family history of diabetes. At this point in the screening process, some participants drew attention to their perceived lack of risk factors such as not having a sweet tooth, whereas others downplayed risk factors such as being overweight. Some participants did not know why they had been invited.

Most participants who tested positive on the first occasion reported being "not unduly worried." A high random blood glucose concentration was often attributed to the food consumed for breakfast or the previous evening, or a healthy fluctuation. Participants typically reported expecting the next (fasting) test to be negative. Indeed the participants interviewed after the first test all said they were not worried. Accounts of the health professionals' reassuring manner in giving results, particularly their use of the term borderline, seemed to contribute to this lack of concern in some cases.

\section{Prediagnostic test expectations}

After testing positive at the second (fasting) test some participants still expected to test negative at the oral glucose tolerance test, one hypothesising that the large number of patients referred for this test meant that only a few would be diabetic. Others had moved to accepting the possibility of type 2 diabetes, albeit a "mild" easily controlled type, often justifying this belief on the absence of symptoms.

All but one participant interviewed before their oral glucose tolerance test seemed to have taken in information about type 2 diabetes from the media or from health professionals, friends, and family at this stage. Some participants (without a family history) had identified people with type 2 diabetes within their own 
Box 3 Typical perceptions at initial stages of the screening process

\section{Screening is good}

Patient 8 (male, impaired glucose tolerance, age 64) "I didn't really think too much about it 'cause l'm a great believer in preventive medicine if you like. It's like preventive maintenance on the car 'cause if you do it beforehand it saves you a lot of problems later down the line"

Patient 102 (female, random blood glucose only, age 69) "I mean it's only been a pin prick up to now hasn't it?"

\section{Expectations of initial test results}

Patient 2 (male, type 2 diabetes, age 67) "I honestly thought l'd have a clear, I can't remember ever suffering from anything, effects of diabetes or anything. I'm grossly overweight but apart from that"

Patient 17 (female, normal, age 58) "I thought l'll just go along and l'd no reason to think there might be anything [diabetes] ... I've never had a particularly sweet tooth"

Patient 4 (female, type 2 diabetes, age 58) "I had been given these tests before from my doctor, because of my family. My grandmother and grandfather both had diabetes and nine out of their 11 children had it, including my mother. And my cousins have got it so I would not be surprised if-it wouldn't be a shock anyway"

\section{Reflections on reason for being invited}

Patient 11 (male, type 2 diabetes, age 55) "I suppose the criteria they put forward was over 40 and overweight... I certainly unfortunately fit into that"

Patient 3 (male, normal, age 69) "No. No. I don't know why I was invited, I think maybe it's because l've had hypertension 'cause they say that it can lead to diabetes, I don't know"

Patient 103 (male, random blood glucose only, 53) "It just said in the letter that I was just picked at random"

\section{Unimportant event}

Patient 3 (male, normal, age 69) "I know it [random blood glucose] was quite high. But l'd had rather a big meal the night before [...] a great big plate of ice cream and two bananas which I imagine put the blood sugar up quite a lot"

Patient 5 (male, impaired glucose tolerance, age 69) "I was surprised at that initial test, that it was higher than the ones l've been doing here, but I thought, well these things happen. And I know with my wife at times her readings do fluctuate"

Patient 1 (male, type 2 diabetes, age 61) "[The nurse] said, 'You'll probably be quite all right but you're on the borderline so we'll get you back just in case'"

\section{Prediagnostic test expectations}

Patient 2 (male, type 2 diabetes, age 67) "I've set my mind that I will probably fail (be diagnosed) tomorrow. But if you catch it early enough you can probably get rid of most of it just by dieting or looking after [yourself]"

Patient 1 (male, type 2 diabetes, age 61) "If I have got diabetes or any form of diabetes, it's very light anyway, you can control it quite easily. It's not-I don't think for one minute l've got it life threatening. I would be dead by now wouldn't I?"

Patient 8 (male, impaired glucose tolerance, age 64) "My cousin's wife went along [...] but it was off the Richter scale. I mean [her blood glucose level] was like 31 for Christ's sake. And even now with it controlled ... it's about 13. I said, "No I was something like 7.1 I think or 7"

Patient 6 (male, impaired fasting glucose, age 50) "Obviously I hope I'm not diabetic and have to inject myself. l've got a couple of friends who are diabetic that do that. But it doesn't seem to have slowed them up too much or worry them"

networks, sometimes using them as a benchmark against which to make favourable comparisons. Others reported how diabetes did not seem to affect their friends' lives, furthering positive perceptions of it being a controllable condition.

\section{Reactions after diagnosis (box 4)}

\section{Nerwly diagnosed type 2 diabetes}

The most common reaction to being diagnosed with type 2 diabetes was to downplay its importance; only one participant reported shock. Testing positive at the first two tests seemed to lead participants to adjust their expectations from testing negative to an increased likelihood of having diabetes. A few participants reported symptoms, previously not considered relevant (such as tiredness and thirst), that they now linked to type 2 diabetes.

The one participant to describe shock was also afraid about the severe consequences of type 2 diabetes. In contrast, the rest emphasised the lack of severity they associated with the disease. All newly diagnosed patients talked confidently about their plans to control the disease; in some cases a diet-only regimen fuelled the perception that their diabetes was mild. Furthermore, most of this group reported being grateful that the screening programme had identified their diabetes at a treatable stage; indeed one patient described it as "a wake up call" to change his lifestyle.

\section{Intermediate and negative results}

Participants with intermediate (impaired fasting glucose or impaired glucose tolerance) or negative oral glucose tolerance test results suggested that they had known they did not have diabetes despite their earlier high readings. Some stated that they would have been surprised if they had been diagnosed, which contrasts with the lack of surprise reported by those who were. Often this belief was reinforced by lack of symptoms, despite being apparently aware of the disease's early asymptomatic period.

Many participants diagnosed with an intermediate condition seemed confused. They appeared to be unaware of this diagnostic label or struggled to explain its meaning, or had received seemingly confused messages from their general practitioner. Most patients seemed unconcerned by their result, often normalising the condition, and reported feeling reassured by their general practitioner or nurse who had recommended simply annual checks. This diagnosis had not triggered lifestyle change even in those who had expressed intentions to change if diagnosed with type 2 diabetes in the pretest interview. For example, one patient said before his oral glucose tolerance test, "One knows there's a chance of it and I think one can then say, right, well if that's the case how do I deal with it and try and take sensible precautions." But after diagnosis he said, "And so I was relieved not to have to have something else to worry about."

Only one participant, unhappy with his general practitioner's explanation, wanted further information about impaired glucose tolerance. Participants diagnosed with intermediate conditions had mixed views about their likelihood of getting type 2 diabetes in the future. Some patients accepted that lifestyle change would affect their risk of developing diabetes, but none appeared to be aware of the risk of cardiovascular disease associated with impaired fasting glucose or impaired glucose tolerance. 


\section{Box 4 Reaction to diagnosis}

\section{Type 2 diabetes}

Patient 9 (female, age 58) "Last time I saw you when we'd done the first interview, I think I was quite sort of blasé ... since l've been diagnosed I'm trying to get my head around it, and l'm finding it difficult. [. . .] To put it plainly l'm scared"

Patient 10 (male, age 66) "I rather suspected that once having got as far as having to go to [hospital], that [type 2 diabetes] was gonna be the outcome"

Patient 16 (female, age 63) "And I think there's-that was where the tell-tale sign that I had trouble ... [my husband] used to say to me, 'you drink too much bloomin' water"' Patient 10 (male, age 66) "It's not like you're being told you've got cancer, it's only diabetes for goodness sake innit? I mean, I must admit that everybody else seems to be taking it much more seriously than I am"

Patient 2 (male, age 67) "He [general practitioner] explained that people call it a mild form of the actual thing. But it wasn't mild, that was wrong, it was a type. And there was two ways of controlling it which was either tablets or diet, and they decided to go on the diet this time with me"... "and if that don't work well obviously it's medication. But ... I'm quite confident that in myself that the diet will control it"

Patient 1 (male, age 61) "If it wasn't for [ADDITION] it wouldn't have been picked up when it was, which means in a few years time I could be in some mess and it would be far too late to do anything then"

\section{No diabetes}

Patient 3 (male, normal, age 69) “I didn't really think that l'd got diabetes, 'cause I mean you can usually get symptoms don't you?"

Patient 13 (male, impaired fasting glucose, age 69) "There was one little glitch where something showed up and it was very technical, about how quickly the blood can absorb sugar or something"

Patient 14 (male, impaired glucose tolerance, age 69) "I come out okay, well I come out - I come out with glucose intolerance, glucose impaired tolerance, which is below, right? It means you're not diabetic but you could be-if you go down the path of-you know-that will bring you to it. And lots of people have got it haven't they?"

Patient 15 (male, impaired glucose tolerance, age 53) "He [general practitioner] said, 'Yeah, you're fine, no problem ... You've got a slight intolerance to glucose ... No problem, you're just like the man next door.' I said, 'Yeah, but it all depends on what the man next door's like doesn't it?"”

Patient 5 (male, impaired glucose tolerance, age 69) "I suppose depending upon what I eat and do and that sort of thing. I suppose that's the things that affect it and ... I suppose could tip it over into being a positive reading then"

\section{DISCUSSION}

This prospective qualitative study highlights the fluid and changing nature of participants' perceptions at different stages of a stepwise screening programme for type 2 diabetes. The data indicate that participants underwent a process of psychological adjustment as they progressed through the programme. The findings help to explain the low levels of anxiety seen among participants in the ADDITION (Cambridge) screening programme, including those eventually diagnosed with the disease. ${ }^{6}$

Although participants talked about diabetes screening being a good thing - enabling the disease to be detected at an early, supposedly treatable stage - on a personal level most tended to downplay or not engage with their individual risk. Many participants, for instance, talked about attending the first test without considering the possibility of testing positive. Upon receipt of a positive test, there was a tendency to use an explanation other than diabetes and expect the next test to be negative.

By the time of the final diagnostic (oral glucose tolerance) test, participants had had time (nine to
10 weeks) to take in diabetes related information from the media or through conversations with friends, family, or health professionals. They had typically moved to accepting the increased possibility of being diagnosed with type 2 diabetes, but of a mild and controllable type. Minimising the threat of type 2 diabetes in this way may have helped participants prepare themselves mentally for a positive diagnosis, and helps account for the lack of anxiety seen in the participants who reached this stage of the programme. ${ }^{6}$

After diagnosis, the confidence that those diagnosed with type 2 diabetes expressed in their plans to control their disease seemed to be related to several perceptions and factors: the disease having been discovered "at an early stage", a lack of severe complications, ${ }^{16}$ and being on a diet-only regimen..$^{10}$ Our findings suggest that the duration and stepwise nature of the screening process is also salient, enabling gradual psychological adjustment. However many stages there are in a screening programme, people will have to go through a process of readjusting their expectations of personal risk. In the absence of a stepwise screening process, psychological reactions might be different. Hence, future research should compare the effect of a stepwise screening programme with a one-off diagnostic oral glucose tolerance test.

\section{Patients with intermediate and negative results}

Participants with impaired fasting glucose or impaired glucose tolerance tended to lack awareness of this diagnosis or struggled to explain the meaning and its implications. These participants, and those with a negative final test result, also expressed no intentions to change their lifestyles, despite having high blood glucose concentrations in the first few tests and the increased cardiovascular risk associated with impaired glucose tolerance or impaired fasting glucose. Given that many participants had not realised about these risks earlier in the screening process, this finding is unsurprising. It may also indicate a lack of accepted professional understanding and management protocols for treating patients at increased metabolic and cardiovascular risk, a problem previously raised by a qualitative study with general practitioners. ${ }^{17}$

\section{Implications}

The findings have important implications for people who implement screening. Even patients who tested negative at the first two tests remain at high risk of developing type 2 diabetes (as the risk score identified them in the top quarter for risk). ${ }^{12}$ Hence, the minimal importance attached to the first test, and relief arising from a negative random or fasting blood glucose test, could undermine the population benefit of a screening programme if these people do not realise that their risk remains high. ${ }^{18}$ Thus, patients should be made aware of the risk factors that led to their screening invitation. Furthermore, the lack of intentions to change lifestyle in participants who did not test positive at the final diagnosis raises questions of when and how "risk of 


\section{WHAT IS ALREADY KNOWN ON THIS TOPIC}

- Quantitative studies have reported a limited psychological effect of screening for type 2 diabetes

- Qualitative work has shown that patients with screen detected type 2 diabetes tend to think their disease is not serious

\section{WHAT THIS STUDY ADDS}

- Participants' perceptions of type 2 diabetes and their risk of developing the disease changed over the course of a diabetes screening programme

- The stepwise nature of the screening programme seemed to facilitate psychological adjustment

- Participants were uncertain about the meaning of intermediate screening results, and those with negative results were unaware they remained at high risk

diabetes" might be effectively conveyed to patients to motivate changes in lifestyle. The interviews carried out before the oral glucose tolerance test indicate that this stage might be a useful point at which to give patients information about the consequences of a positive or negative test, so that those not diagnosed with type 2 diabetes are made aware of their risk of developing diabetes and cardiovascular disease.

The lack of anxiety associated with the screening programme may suggest a low psychological cost to implementing screening nationally. However, the tendency not to perceive type 2 diabetes as a serious condition is a potential concern. The information conveyed to participants about their screening result is key to their understanding. ${ }^{19}$ A challenge for health professionals is to convey enough information about the potential consequences of the disease to justify lifestyle change, without raising anxiety sufficiently to cause disengagement. ${ }^{7}$ The ADDITION (Cambridge) trial produced protocols outlining the form of words to be used when giving out results, but without observing the tests it is impossible to know what was actually said. Hence, future research could look at diagnostic consultations between health professionals and people with screen detected type 2 diabetes and impaired fasting glucose or impaired glucose tolerance.

\section{Strengths and limitations of the study}

The study was strengthened by its prospective component, enabling investigation of participants' views before and after their final diagnosis. An additional strength is its focus on reactions to intermediate results as well as positive and negative diagnostic results. The study's generalisability may be limited by its reliance on an opt-in procedure and low response rate. However, while it is impossible to establish whether participants were more or less anxious about their health than non-participants, the findings concur with previous literature ${ }^{10}$ and the larger quantitative study. ${ }^{6}$

We are grateful to the participants for their time and involvement. We thank the ADDITION (Cambridge) trial coordination team, in particular Kate Williams (trial manager); the practices; the healthcare professionals; and the MRC field epidemiology team who helped with recruitment. Interviews were transcribed by Academic Transcriptions (Cambridge).

Contributors: The study was conceived and designed by all authors. HE coordinated the study and conducted all interviews. HE and RD analysed and interpreted the data with supervision from JL. HE wrote the first draft of the paper with input from JL and RD. All authors contributed to the final draft. HE is guarantor.

Funding: This study was funded by a grant from the Royal College of General Practitioners scientific foundation board (SFB/2005/12). The ADDITION (Cambridge) trial was funded by the Wellcome Trust (G0000753). HE is funded by an ESRC/MRC post doctoral fellowship.

Competing interests: None declared.

Ethical approval: Eastern medical research ethics committee (02/5/54).

1 Harris MI, Klein R, Welborn TA, Knuiman MW. Onset of NIDDM occurs at least 4-7 years before clinical diagnosis. Diabetes Care 1992;15:815-9.

2 WHO. Definition, diagnosis and classification of diabetes mellitus and its complications. WHO/NCD/NCS/99.2. Geneva: WHO Department of Noncommunicable Disease Surveillance, 1999.

3 Colagiuri S, Cull CA, Holman RR. Are lower fasting plasma glucose levels at diagnosis of type 2 diabetes associated with improved outcomes? UK prospective diabetes study 61. Diabetes Care 2002;25:1410-7.

4 Diabetes Prevention Program Research Group. Reduction in the incidence of type 2 diabetes with lifestyle intervention or metformin. N Engl J Med 2002;346:393-403.

5 Lauritzen T, Griffin S, Borch-Johnsen K, Wareham N, Wolffenbuttel BHR, Rutten G. The ADDITION study: proposed trial of the costeffectiveness of an intensive multifactorial intervention on morbidity and mortality among people with type 2 diabetes detected by screening. Int J Obes 2000;(suppl 3):S6-11.

6 Eborall HC, Griffin SG, Prevost AT, Kinmonth AL, French DP, Sutton SR. Psychological impact of screening for type 2 diabetes: controlled tria and comparative study embedded in the ADDITION (Cambridge) randomised controlled trial. BM/ doi: 10.1136 bmj.39303.723449.55

7 Peel E, Parry O, Douglas M, Lawton J. Diagnosis of type 2 diabetes: a qualitative analysis of patients' emotional reactions and views about information provision. Patient Educ Couns 2004;53:269-75.

8 Parry O, Peel E, Douglas M, Lawton J. Patients in waiting: a qualitative study of type 2 diabetes patients' perceptions of diagnosis. Fam Pract 2004;21:131-6.

9 Carroll C, Naylor E, Marsden P, Dornan T. How do people with type 2 diabetes perceive and respond to cardiovascular risk? Diabet Med 2002;20:355-60.

10 Adrianse MC, Snoek FJ, Dekker JM, Van der Ploeg HM, Heine RJ. Screening for type 2 diabetes: an exploration of subjects perceptions regarding diagnosis and procedure. Diabet Med 2002;19:406-11.

11 Britten N, Jones R, Murphy E, Stacy R. Qualitative research methods in general practice. Fam Pract 1995;12:104-14.

12 Glaser B, Strauss A. Thediscovery of grounded theory. Chicago: Aldine Publishing Company, 1967.

13 Strauss A, Corbin J. Basics of qualitative research: grounded theory procedures and techniques. London: Sage, 1990.

14 Griffin SJ, Little PS, Kinmonth AL, Hales CN, Wareham NJ. Diabetes risk score: towards earlier detection of type 2 diabetes in general practice. Diabetes Metab Res Rev 2000;16:164-71.

15 World Health Organization. Diabetes mellitus: report of a WHO study group. Geneva: WHO, 1985.

16 Murphy E, Kinmonth AL. No symptoms, no problem? Patients understandings of non-insulin dependent diabetes. Fam Pract 1995;12:184-92.

17 Wylie G, Hungin APS, Neely J. Impaired glucose tolerance: qualitative and quantitative study of general practitioners' knowledge and perceptions. BM/ 2002;324:1190-5.

18 Marteau TM, Kinmonth AL, Thompson S, Pyke S. The psychological impact of cardiovascular screening and intervention in primary care: a problem of false reassurance? Br J Gen Pract 1996;46:577-82.

19 Shaw C, Abrams K, Marteau TM. Psychological impact of predicting individuals' risks of illness: a systematic review. Soc Sci Med 1999;49:1571-98.

Accepted: 10 August 2007 